\title{
Matrix Representations for Positive Noncommutative Polynomials
}

\author{
J.William Helton * $\quad$ Scott A. McCullough ${ }^{\dagger} \quad$ Mihai Putinar ${ }^{\ddagger}$ \\ September 17, 2003
}

\begin{abstract}
In real semialgebraic geometry it is common to represent a polynomial $q$ which is positive on a region $R$ as a weighted sum of squares. Serious obstructions arise when $q$ is not strictly positive on the region $R$. Here we are concerned with noncommutative polynomials and obtaining a representation for them which is valid even when strict positivity fails.

Specifically, we treat a "symmetric" polynomial $q(x, h)$ in noncommuting variables, $\left\{x_{1}, \ldots, x_{g_{x}}\right\}$ and $\left\{h_{1}, \ldots, h_{g_{h}}\right\}$ for which $q(X, H)$ is positive semidefinite whenever

$$
X=\left(X_{1}, \ldots, X_{g_{x}}\right) \text { and } H=\left(H_{1}, \ldots, H_{g_{h}}\right)
$$

are tuples of selfadjoint matrices with $\left\|X_{j}\right\| \leq 1$ but $H_{j}$ unconstrained. The representation we obtain is a Gram representation in the variables $h$

$$
q(x, h)=V(x)[h]^{T} P_{q}(x) V(x)[h],
$$

where $P_{q}$ is a symmetric matrix whose entries are noncommutative polynomials only in $x$ and $V$ is a "vector" whose entries are polynomials in both $x$ and $h$. We show that one can choose $P_{q}$ such that the matrix $P_{q}(X)$ is positive semidefinite for all $\left\|X_{j}\right\| \leq 1$. The representation covers sum of square results $([\mathrm{H}],[\mathrm{M}],[\mathrm{MP}])$ when $g_{x}=0$. Also it allows for arbitrary degree in $h$, rather than degree two, in the main result of [CHSY] when restricted to $x$-domains of the type $\left\|X_{j}\right\| \leq 1$.
\end{abstract}

${ }^{*}$ Partially supported by NSF, DARPA and Ford Motor Co.

${ }^{\dagger}$ Partially supported by NSF grant DMS-0140112

${ }^{\ddagger}$ Partially supported by NSF grant DMS-0100367 


\section{Introduction}

Let $\mathcal{N}$ denote the algebra over $\mathbb{R}$ of polynomials in the noncommuting variables $x:=\left\{x_{1}, \ldots, x_{g_{x}}\right\}$ and $h:=\left\{h_{1}, \ldots, h_{g_{h}}\right\}$. An element of $\mathcal{N}$ is thus a real finite linear combination of words in $x$ and $h$ and is called a noncommutative polynomial (abbreviated NC polynomial). Given integers $M$ and $N$, let $\mathcal{N}_{M, N}$ denote the set of noncommutative polynomials in $\mathcal{N}$ which have degree at most $M$ in $x$ and at most $N$ in $h$. For example,

$$
p(x, h)=x_{1} h_{2} x_{3}+5 x_{2} x_{1} h_{2} x_{1}
$$

is a polynomial in $\mathcal{N}_{3,1}$.

The natural involution ${ }^{T}$ on $\mathcal{N}$ defined by

$$
w=z_{1} \ldots z_{n} \mapsto w^{T}=z_{n} \cdots z_{2} z_{1}
$$

for $w$ a word in $\{x, h\}$ and

$$
q=\sum q_{w} w \in \mathcal{N} \mapsto q^{T}=\sum q_{w} w^{T}
$$

fixes $\mathcal{N}_{M, N}$. Here each $z_{j} \in\left\{x_{1}, \ldots, x_{g_{x}}, h_{1}, \ldots, h_{g_{h}}\right\}$. A polynomial $q$ in $\mathcal{N}$ is symmetric provided $q^{T}=q$. For example,

$$
p(x, h)=x_{1} h_{2} x_{3}+5 x_{2} x_{1} h_{1} x_{1}+x_{3} h_{2} x_{1}+5 x_{1} h_{1} x_{1} x_{2}
$$

is a symmetric polynomial in $\mathcal{N}_{3,1}$.

Let $\mathcal{B}(\mathcal{H})$ denote the collection of bounded linear operators on the real Hilbert space $\mathcal{H}$. Given tuples $X=\left(X_{1}, \ldots, X_{g_{x}}\right)$ and $H=\left(H_{1}, \ldots, H_{g_{h}}\right)$ of, not necessarily commuting, selfadjoint operators from $\mathcal{B}(\mathcal{H})$ and $p \in \mathcal{N}$, define $p(X, H) \in \mathcal{B}(\mathcal{H})$ in the natural way by substitution. For instance, for the polynomial $p$ in $(1)$,

$$
p(X, H)=X_{1} H_{2} X_{3}+5 X_{2} X_{1} H_{1} X_{1}+X_{3} H_{2} X_{1}+5 X_{1} H_{1} X_{1} X_{2} .
$$

The main result of this paper is a representation theorem for symmetric polynomials $q \in \mathcal{N}$ such that $q(X, H) \geq 0$ for all tuples $X$ and $H$ of selfadjoint operators on a common Hilbert space, with each $X_{j}$ a contraction, $\left\|X_{j}\right\| \leq 1$. Here, and throughout the present article, $\|A\|$ refers to the operator norm of $A \in \mathcal{B}(\mathcal{H})$ and $A$ positive semidefinite, denoted $A \geq 0$, means $A=A^{T}$ and $\langle A h, h\rangle \geq 0$ for all $h \in \mathcal{H}$.

The evaluation map $p \in \mathcal{N} \mapsto p(X, H) \in \mathcal{B}(\mathcal{H})$ determines a mapping from $M_{n}(\mathcal{N})$, the $n \times n$ matrices with entries from $\mathcal{N}$, into $\mathcal{B}\left(\oplus_{1}^{n} \mathcal{H}\right)=$ 
$M_{n}(\mathcal{B}(\mathcal{H}))$ by evaluating entry-wise. Thus, if $M=\left(m_{j, k}\right) \in M_{n}(\mathcal{N})$, then $M(X, H)=\left(m_{j, k}(X, H)\right)$.

All the definitions and the notation make sense in the case $g_{h}=0$, that is, where the polynomials depend on the variable $x$ only. In this case we write $\mathcal{N}_{x}$ instead of $\mathcal{N}$. The involution ${ }^{T}$ extends to matrix polynomials $M=\left(m_{j, k}\right)_{j, k=1}^{n} \in M_{n}\left(\mathcal{N}_{x}\right)$ as $M^{T}=\left(m_{k, j}^{T}\right)_{j, k=1}^{n}$ and $M$ is symmetric if $M^{T}=M$. In the case $M(x)$ is symmetric, we say $M$ is semipositive (resp. semipositive on the noncommutative polydisk) if $M(X) \geq 0$ for all tuples $X$ of selfadjoint operators (resp. for selfadjoint contractions, $\left.\left\|X_{j}\right\| \leq 1\right)$.

A symmetric polynomial $q \in \mathcal{N}$ has a Gram Representation in $h$

$$
q(x, h)=V(x)[h]^{T} P_{q}(x) V(x)[h],
$$

where the tautological vector $V(x)[h]$ has the form

$$
V(x)[h]=\left(\begin{array}{c}
e \\
h_{1} w_{1}^{1} \\
\vdots \\
h_{1} w_{\ell_{1}}^{1} \\
\vdots \\
h_{k} w_{1}^{k} \\
\vdots \\
h_{k} w_{\ell_{k}}^{k}
\end{array}\right) .
$$

Here $w_{j}^{i} \in \mathcal{N}, e=\emptyset$ is the identity for $\mathcal{N}$, and $P_{q}$ is a symmetric matrix whose entries are noncommutative polynomials in $x$.

For example, the polynomial $p$ from (1) has the Gram representations in $h$,

$$
p=\left(\begin{array}{lll}
e & x_{3} h_{2} & x_{1} h_{1}
\end{array}\right)\left(\begin{array}{ccc}
0 & x_{1} & 5 x_{2} x_{1} \\
x_{1} & 0 & 0 \\
5 x_{1} x_{2} & 0 & 0
\end{array}\right)\left(\begin{array}{c}
e \\
h_{2} x_{3} \\
h_{1} x_{1}
\end{array}\right)
$$

and

$$
p=\left(\begin{array}{llll}
e & x_{3} h_{2} & x_{1} h_{1} & x_{1} h_{1}^{2}
\end{array}\right)\left(\begin{array}{cccc}
0 & x_{1} & 5 x_{2} x_{1} & -x_{1} \\
x_{1} & 0 & 0 & 0 \\
5 x_{1} x_{2} & 0 & 2 & 0 \\
-x_{1} & 0 & 0 & 0
\end{array}\right)\left(\begin{array}{c}
e \\
h_{2} x_{3} \\
h_{1} x_{1} \\
h_{1}^{2} x_{1}
\end{array}\right) .
$$

Note that if there is a $P_{q}$ for which $P_{q}(X)$ is semipositive for all $X$ in the noncommutative polydisk, then $q(X, H) \geq 0$ for all tuples $X$ and $H$ 
of selfadjoint operators on a common Hilbert space for which each $X_{j}$ is a contraction, $\left\|X_{j}\right\| \leq 1$. A corollary of our main theorem is the converse.

Theorem 1.1 Suppose $q(x, h)$ is a symmetric NC polynomial in the variables $x$ and $h$. If $q(X, H) \geq 0$ for all selfadjoint tuples $X=\left(X_{1}, \ldots, X_{g_{x}}\right)$ and $H=\left(H_{1}, \ldots, H_{g_{h}}\right)$ acting on finite dimensional (real) Hilbert space where each $X_{j}$ is a contraction, $\left\|X_{j}\right\| \leq 1$, then $q$ has the Gram representation (2) with a symmetric $P_{q}$ which is semipositive on the noncommutative polydisk.

Our main result, Theorem 2.2 refines Theorem 1.1 above by adding precise degrees for the factors in the Gram representation in $h$. Its proof uses a Hahn-Banach separation argument and a Gelfand Naimark Segal type construction $^{1}$ similar to that found in the proof of the (commutative) Positivstellensatz of [PV].

In the special case where $q$ does not depend on $x$, Theorem 1.1 says that every semipositive noncommutative polynomial has the form

$$
q(h)=V[h]^{T} P V[h]
$$

for some positive semidefinite matrix $P$ not depending on $x$. A positive semidefinite matrix $P$ can be factored as $P=L^{T} L$ which yields that $q$ can be written as a sum of squares. Thus Theorem 1.1 yields results much like those in $[\mathrm{H}],[\mathrm{M}]$, and $[\mathrm{MP}]$.

When $q(x, h)=Q(x) \in M_{n}\left(\mathcal{N}_{x}\right)$ is a matrix valued polynomial which does not depend upon $h$, the NC Positivstellensatz in [HM] says that if $Q$ is strictly positive on the polydisk, $Q(X)>0$ for all tuples $X$ of selfadjoint contractions, then $Q$ has a weighted sum of squares (SoS) representation. Indeed, this NC Positivstellensatz is key in the proof of Theorem 1.1. In fact, there are many noncommutative domains which work equally as well as the noncommutative polydisk. For instance, $Q(X, H)$ is strictly positive on the noncommutative ball if $Q(X, H)>0$ whenever $X$ is a tuple of selfadjoint operators satisfying $I-\sum X_{j}^{2} \geq 0$ and $H$ is a tuple of selfadjoint operators. If $P(X)$ is strictly positive on the noncommutative ball, then $P$ has a weighted sum of squares representation and the arguments in this paper show that if $Q(X, H)$ is strictly positive on the noncommutative ball, then $Q=V^{T}(x)[h] P(x) V(x)[h]$ for some $P$ which is positive semidefinite on the noncommutative ball.

\footnotetext{
${ }^{1}$ this represents an abstract $C^{*}$-algebra as an algebra of bounded operators on a Hilbert space.
} 
While we emphasize that strict positivity makes behavior nicer and is required in general in $\mathrm{NC}$ possitivstellensatz, there are situations where it is not required. A tuple $X=\left(X_{1}, \ldots, X_{g_{x}}\right)$ is a spherical isometry if $\sum X_{j}^{T} X_{j}=I$, where the $X_{j}$ are not necessarily selfadjoint, and the polynomials below are polynomials in both $x_{j}$ and $x_{j}^{T}$. The result [HMP] says, if $P(X) \geq 0$ for all $\mathrm{X}$ which are spherical isometries, then there is a NC polynomial $S$ which is a SoS of polynomials such that

$$
P(X)=S(X) \text { for all spherical isometries } X \text {. }
$$

Thus, results in this paper and [HMP] suggest, if $q(X, H)$ is positive semidefinite whenever $X$ is a spherical isometry and $H$ is arbitrary, then $q=s+r$, where $s$ is a sum of squares and $r$ residual part $r(x, h)$ which vanishes on spherical isometries, that is, $r(X, H)=0$ when $X$ is a spherical isometry so that $q(X, H)=s(X, H)$.

Returning to the mixed case, when $q(x, h)$ is semipositive on the noncommutative polydisk and homogeneous of degree two in $h$, Corollary 1.1 contains a major piece of the principal result of [CHSY].

\section{Notation and Main Result}

Before stating the main result, we first formalize the notation used in the introduction.

\section{$2.1 \quad$ Notation}

Since the $x$ and $h$ variables play asymmetric roles, they are treated separately, rather than simply considering $g_{x}+g_{h}$ noncommutative variables. Let $\mathcal{F}$ denote the free semigroup on the alphabet $\{x, h\}=\left\{x_{1}, \ldots, x_{g_{x}}, h_{1}, \ldots, h_{g_{h}}\right\}$, that is, all words in these letters. The empty word, $\emptyset$, plays the role of the multiplicative identity, as $\emptyset w=w \emptyset=w$ for $w \in \mathcal{F}$. For given nonnegative integers $M$ and $N$, let $\mathcal{F}_{M, N}$ denote words in these variables of length at most $M$ in $x$ and $N$ in $h$.

The noncommutative polynomials $\mathcal{N}$ can be thought of as the free semigroup real algebra on the alphabet $\{x, h\}$. Concretely, an element $p$ of $\mathcal{N}$ is an expression of the form,

$$
p=\sum_{w \in \mathcal{F}} p_{w} w,
$$

where the sum is finite, and is called a polynomial, or NC polynomial, in $\{x, h\}$. The empty word is the multiplicative identity and the empty sum, 0 , 
is the additive identity for $\mathcal{N}$. Let $\mathcal{N}_{M, N}$ denote the real vector space with basis $\mathcal{F}_{M, N}$. Equivalently, $\mathcal{N}_{M, N}$ is the subset of $\mathcal{N}$ consisting of those $p$ as in (4) where the sum is over words $w \in \mathcal{F}_{M, N}$.

Let

$$
\Gamma_{M, N}=\Gamma=\{\emptyset\} \cup\left(\cup_{j=1}^{g_{h}} h_{j} \mathcal{F}_{M, N-1}\right) .
$$

That is, $\Gamma$ consists of the empty word and those words in $\mathcal{F}_{M, N}$ which start with some $h_{j}$. Let $|\Gamma|$ denote the cardinality of $\Gamma$. We will use $\Gamma$ as an index set. For example, let $\mathcal{N}_{M, N}^{\Gamma}$ denote the collection of vectors of length $|\Gamma|$ with entries from $\mathcal{N}_{M, N}$, so that an element $W \in \mathcal{N}_{M, N}^{\Gamma}$ is a function $W: \Gamma \mapsto \mathcal{N}_{M, N}$ thought of as a column vector where the $w$-th entry is $W_{w}$, for $w \in \Gamma$.

The tautological vector $V_{M, N}=V=V(x)[h]$ of (3) (see [CHSY], where it is called the border vector ), which plays a key role in our Gram representation in $h$, is the element of $\mathcal{N}_{M, N}^{\Gamma}$ whose $w \in \Gamma$ entry is $w$. Here we use $V$ to denote $V_{M, N}$ with the choice of $M, N$ understood from the context. In what follows, it will be convenient to decompose $V_{M, N}$ as

$$
V_{M, N}=\oplus_{j=0}^{N} V_{M, N}^{j}=\oplus_{j=0}^{N} V^{j},
$$

where each $V^{j}$ is homogeneous of degree $j$ in $h$. For instance, $V^{0}=(\emptyset)$ and $V^{1}$ consists of vectors of the form (3) with all words $w_{i}^{\mu}$ independent of $h$ and without the $e=\emptyset$ term, that is, $w_{i}^{\mu}$ is in $\mathcal{F}_{M, 0}$, and $V$ contains no $e$ term. Thus, with $\Gamma_{0}=\{\emptyset\}$ and

$$
\Gamma_{j}=\left\{h_{\ell} v: 1 \leq \ell \leq g_{h}, \quad v \text { has degree } j-1 \text { in } h\right\},
$$

the vector $V^{j}$ can be viewed as either the element of $\mathcal{N}_{M, N}^{\Gamma_{j}}$ with $w \in \Gamma_{j}$ entry equal to $w$; or as the element of $\mathcal{N}_{M, N}^{\Gamma}$ with $w$-entry $w$ if $w$, has degree $j$ in $h$ and 0 otherwise.

Let $M_{\Gamma}\left(\mathcal{N}_{M, N}\right)$ denote the collection of $|\Gamma| \times|\Gamma|$ matrices with entries from $\mathcal{N}_{M, N}$ indexed by $\Gamma$. Explicitly, $P \in M_{\Gamma}\left(\mathcal{N}_{M, N}\right)$ is a $|\Gamma| \times|\Gamma|$ matrix with $\left(h_{\ell} \alpha, h_{m} \beta\right)$ entry $P_{h_{\ell} \alpha, h_{m} \beta}$ for $1 \leq \ell, m \leq g_{h}$ and $\alpha, \beta \in \mathcal{F}_{M, N-1}$, $\left(\emptyset, h_{m} \beta\right)$ entry $P_{\emptyset, h_{m} \beta}$ for $1 \leq m \leq g_{h}$ and $\beta \in \mathcal{F}_{M, N-1}$, and $P_{\emptyset, \emptyset}$ the $(\emptyset, \emptyset)$ entry. Let $P^{j, j}$ denote the submatrix $\left(P_{h_{\ell} \alpha, h_{m} \beta}\right)$ over those $\alpha, \beta \in$ $\mathcal{F}_{M, N-1}$ which have degree precisely $j-1$ in $h$; here $h_{\ell}$ and $h_{m}$ range over all possibilities. Let $P^{0,0}=P_{\emptyset, \emptyset}$. Thus, if we let $n_{j}$ denote the cardinality of the set of $\alpha \in \mathcal{F}_{M, N-1}$ of degree $j-1$ in $h$, then $P^{j, j}$ is an $n_{j} \times n_{j}$ matrix with entries $P_{\alpha, \beta}^{j, j}$, where $\alpha, \beta$ are of the form $\alpha=h_{m} \alpha^{\prime}, \beta=h_{\ell} \beta^{\prime}$ for some $\ell, m$ or empty $h_{\ell}, h_{m}$ and $\alpha^{\prime}, \beta^{\prime} \in \mathcal{F}_{M, N-1}$ of degree exactly $j-1$ in $h$. In 
a similar manner, define $P^{j, k}$ for $j \neq k$. With these definitions we have, for any $P$ in $M_{\Gamma}$,

$$
\begin{aligned}
V^{T} P V= & \sum_{j, k}\left(V^{j}\right)^{T} P^{j, k} V^{k} \\
= & \sum_{\ell, m} \sum_{\alpha, \beta \in \mathcal{F}_{M, N-1}} \alpha^{T} h_{\ell} P_{h_{\ell} \alpha, h_{m} \beta} h_{m} \beta+\sum P_{\emptyset, h_{m} \beta} h_{m} \beta \\
& +\sum \alpha^{T} h_{\ell} P_{h_{\ell} \alpha, \emptyset}+P_{\emptyset, \emptyset},
\end{aligned}
$$

where $V=V_{M, N}$.

Definition 2.1 Let $\mathcal{P}_{M, N}$ denote those $P \in M_{\Gamma}\left(\mathcal{N}_{x}\right)$ such that

1. $P(X) \geq 0$ for each tuple $X=\left(X_{1}, \ldots, X_{g_{x}}\right)$ of (not necessarily commuting) contractions on a (common) Hilbert space $\mathcal{H}$;

2. $P_{h_{\ell} \alpha, h_{m} \beta}$ has degree at most $2 M$ minus the sum of the degrees of $\alpha$ and $\beta$ in $x$ for $h_{\ell} \alpha, h_{m} \beta \in \Gamma$ so that the degree of $\alpha^{T} h_{\ell} P_{h_{\ell} \alpha, h_{m} \beta} h_{m} \beta$ is at most $2 M$ in $x$; and

3. $P_{h_{\ell} \alpha, \emptyset}$ and $P_{\emptyset, h_{m} \beta}$ have degree at most $2 M$ minus the degree of $\alpha$ in $x$ and $2 M$ minus the degree of $\beta$ in $x$ respectively, and $P_{\emptyset, \emptyset}$ has degree at most $2 M$.

Let $\mathcal{C}_{M, N}$ denote the set of polynomials with a Gram representation in $h$ of appropriate dimension, namely,

$$
\mathcal{C}_{M, N}=\left\{V_{M, N}^{T} P V_{M, N}: P \in \mathcal{P}_{M, N}\right\}
$$

and let $\mathcal{C}$ denote the union of all the $\mathcal{C}_{M, N}$. Note that if $q \in \mathcal{C}$ and $X$ and $H$ are tuples of selfadjoint operators on a real Hilbert space and each $X_{j}$ is a contraction, then $q(X, H) \geq 0$.

\subsection{Main result}

Theorem 2.2 Suppose $q(x, h)$ is a symmetric NC polynomial in the selfadjoint variables $x$ and $h$. If $q(X, H) \geq 0$ for all selfadjoint tuples $X=$ $\left(X_{1}, \ldots, X_{g_{x}}\right)$ and $H=\left(H_{1}, \ldots, H_{g_{h}}\right)$ acting on a finite dimensional (real) Hilbert space where each $X_{j}$ is a contraction, $\left\|X_{j}\right\| \leq 1$, then $q \in \mathcal{C}$. In fact, if $q \in \mathcal{N}_{M+1,2 N}$, then $q \in \mathcal{C}_{M+1, N+1}$. 
With some additional care, an upper bound on the dimension of the Hilbert spaces required in Theorem 2.2 can be given.

We note that, in the case that item (1) of Definition 2.1 is replaced by $P(X)>0$ there is a representation for $P$, found in [MP], much like Stengle's Positivstellensatz in the commutative case $[\mathrm{St}]$.

\section{Components of the proof}

\subsection{Some first words about words}

Given a polynomial $r=\sum_{v} r_{v} v \in \mathcal{N}_{M, N}$, a word $u$ appears in $r$ provided $r_{u} \neq 0$.

Lemma 3.1 If $\alpha, \beta, \gamma, \delta$ are words of degree $j-1$ in $h$, if $v, w$ are words in $x$ only, and if

$$
\beta^{T} h_{\ell} v h_{k} \alpha=\delta^{T} h_{n} u h_{m} \gamma,
$$

then $\alpha=\gamma, \beta=\delta, \ell=n, k=m$, and $u=v$. In particular, if $\left(h_{k} \alpha, h_{\ell} \beta\right) \neq$ $\left(h_{m} \gamma, h_{n} \delta\right)$, and if $p, q \in \mathcal{N}_{x}$, then the words appearing in $\beta^{T} h_{\ell} p h_{k} \alpha$ are disjoint from those appearing in $\delta^{T} h_{n} q h_{m} \gamma$.

Proof. Without loss of generality, assume that the degree of $\alpha$ is at least as large as that of $\gamma$, so that there is a word $\epsilon$ such that $\alpha=\epsilon \gamma$. Thus,

$$
\beta^{T} h_{\ell} v h_{k} \epsilon=\delta^{T} h_{n} u h_{m} .
$$

Since the degree in $h$ of the polynomial on the right hand side is $j+1$, it follows that $\epsilon$ is a polynomial in $x$ alone. Hence $\epsilon$ is the identity (empty word).

Lemma 3.2 If $p \in \mathcal{N}_{M, N}$, then $p^{T} p \in \mathcal{C}_{M, N}$ and $p^{T}\left(1-x_{k}^{2}\right) p \in \mathcal{C}_{M+1, N}$ for each $1 \leq k \leq g_{x}$.

Proof. Write $p=\alpha_{0}+\sum_{\alpha, k, \beta} p_{\alpha h_{k} \beta} \alpha h_{k} \beta$, where $\alpha_{0}$ and each $\alpha$ is a polynomial in $x$ alone and each $\beta$ is a polynomial in both $x$ and $h$. Let $r_{k, \beta}=\sum_{\alpha} p_{\alpha h_{k} \beta} \alpha$ for $1 \leq k \leq g_{h}$, let $r_{0}=\alpha_{0}$, and let $r$ denote the (row) vector $r=\left(r_{k, \beta}\right)$, indexed by $\Gamma_{M, N}$. Then

$$
\begin{aligned}
r V & =\alpha_{0} e+\sum r_{k, \beta} h_{k} \beta \\
& =\alpha_{0}+\sum_{k, \beta, \alpha} p_{\alpha h_{k} \beta} \alpha h_{k} \beta \\
& =p,
\end{aligned}
$$


where $V$ is the tautological vector of $p$. Now let $R=r^{T} r$. As the degree of $r_{k, \beta} h_{k} \beta$ is at most $M$ in $x$, the degree of $R_{h_{\ell} \delta, h_{k} \beta}=r_{k, \beta}^{T} r_{\ell, \delta}$ is at most $2 M$ minus the sum of the degrees of $\beta$ and $\delta$ in $x$. Further, since $R$ is a square, $R(X) \geq 0$ for all tuples $X$, not just tuples of contractions Thus $R \in \mathcal{P}_{M, N}$. Consequently,

$$
p^{T} p=V^{T} r^{T} r V
$$

is in $\mathcal{C}_{M, N}$. Similarly, $r^{T}\left(1-x_{k}^{2}\right) r$ is in $\mathcal{P}_{M+1, N}$ (although, unlike $R$, it is not necessarily positive semidefinite on all tuples $X$, just tuples of contractions) and so

$$
p^{T}\left(1-x_{k}^{2}\right) p=V^{T} r^{T}\left(1-x_{k}^{2}\right) r V \in \mathcal{C}_{M+1, N} .
$$

\subsection{Positive functionals, tuples, and the openness condition}

In this section we construct, for a given $M$ and $N$, tuples $X$ and $H$ of selfadjoint operators with $X$ contractive, such that the evaluation representation $p \in \mathcal{N}_{M, N} \mapsto p(X, H)$ is faithful; that is, if $p(X, H)=0$ then $p=0$.

Lemma 3.3 Given $M$ and $N$, there exists a linear functional $\lambda: \mathcal{N}_{2 M, 2 N} \rightarrow$ $\mathbb{R}$ such that $\lambda\left(p^{T} p\right)>0$ for all nonzero $p \in \mathcal{N}_{M, N}$.

Proof. As in this setting there is no difference between the variables $x$ and $h$, we may assume that our polynomials are polynomials in $x$ alone. Let $\mathcal{U}_{d}$ denote the collection of polynomials in $x$ of degree at most $d$. Given $d$, it suffices to prove that there exists $\lambda_{2 d}: \mathcal{U}_{2 d} \longrightarrow \mathbb{R}$ such that $\lambda_{2 d}\left(p^{T} p\right)>0$ for all nonzero $p \in \mathcal{U}_{d}$.

The strategy is to construct positive definite "Hankel" inner products, $<\cdot, \cdot\rangle_{d}$ on $\mathcal{U}_{d}$, namely ones with the property that $\langle p, q\rangle_{d}$ is a function of $q^{T} p$ only, for $p$ and $q$ in $\mathcal{U}_{d}$. Once this is done, define $\lambda_{2 d}: \mathcal{U}_{2 d} \longrightarrow \mathbb{R}$ by $\lambda_{2 d}(p)=<p, \emptyset>_{2 d}$ and note, if $p \in \mathcal{U}_{d}$ is not zero, then $p^{T} p \in \mathcal{U}_{2 d}$ and $\lambda_{2 d}\left(p^{T} p\right)=<p^{T} p, \emptyset>_{2 d}=<p, p>_{2 d}>0$.

The construction of the inner products proceeds by induction. We can define $\left\langle c_{1} \emptyset, c_{2} \emptyset>_{0}=c_{1} c_{2}\right.$, on $\mathcal{U}_{0}$, where $c_{1}$, and $c_{2}$ are real constants. Thus the induction starts. Now suppose that $\langle\cdot, \cdot\rangle_{d}$ has been defined. Define $<\cdot, \cdot\rangle_{d+1}$, depending on a positive constant $C$, as follows.

$$
<u, v>_{d+1}= \begin{cases}<u, v>_{d} & \text { if }\left|v^{T} u\right| \leq 2 d \\ 0 & \text { if }\left|v^{T} u\right|=2 d+1 \\ C \delta_{u, v} & \text { if }\left|v^{T} u\right|=2 d+2\end{cases}
$$


where $\delta$ denotes the Kronecker symbol. The induction hypothesis implies $<\cdot, \cdot>_{d+1}$ restricted to $\mathcal{U}_{d}$ is (strictly) positive definite. Hence, there exist a large enough $C$ so that $\langle\cdot, \cdot\rangle_{d+1}$ (strictly) positive definite on $\mathcal{U}_{d+1}$.

Now define $\lambda_{2 d}: \mathcal{U}_{2 d} \longrightarrow \mathbb{R}$ by $\lambda_{2 d}(p)=<p, \emptyset>_{d}$ and note that, for $p \in \mathcal{U}_{d}, \lambda_{2 d}\left(p^{T} p\right)=<p, p>_{d}>0$, as required.

Lemma 3.4 Fix $M, N$ and a linear functional $\lambda: \mathcal{N}_{2(M+1), 2(N+1)} \longrightarrow \mathbb{R}$ such that $\lambda(p)=\lambda\left(p^{T}\right)$. Let $d$ denote the dimension of $\mathcal{N}_{M, N}$.

(a) If $\lambda\left(p^{T} p\right)>0$ for all nonzero $p \in \mathcal{N}_{M+1, N+1}$, then there exists a (real) Hilbert space $\mathcal{H}$ of dimension d with inner product $\langle\cdot, \cdot\rangle$, a vector $\gamma \in \mathcal{H}$, a tuple $X$ of selfadjoint operators on $\mathcal{H}$ and a tuple $H$ of selfadjoint operators on $\mathcal{H}$ such that $<p(X, H) \gamma, r(X, H) \gamma\rangle=\lambda\left(r^{T} p\right)$ for $p, r \in \mathcal{N}_{M, N}$.

(b) If, moreover, $\lambda(p) \geq 0$ for $p \in \mathcal{C}_{M+1, N+1}$, then each $X_{k}$ can be chosen a contraction, $\left\|X_{k}\right\| \leq 1$.

Proof. Let $\mathcal{K}$ denote the Hilbert space obtained by introducing the inner product

$$
<p, q>=\lambda\left(q^{T} p\right)
$$

on $\mathcal{N}_{M+1, N+1}$. The hypothesis on $\lambda$ guarantees there are no null vectors and thus the dimension of $\mathcal{K}$ is $d$ and we may define the following operators. Let $\mathcal{H}$ denote the set $\mathcal{N}_{M, N}$ as a subspace of $\mathcal{K}$ and let $\mathcal{L}$ denote the orthogonal complement of $\mathcal{H}$ in $\mathcal{K}$. Define $\mathbb{X}_{k} p=x_{k} p$ if $p \in \mathcal{H}$ and $\mathbb{X}_{k} p=0$ if $p \in \mathcal{L}$. Similarly define $\mathbb{H}_{k} p=h_{k} p$ if $p \in \mathcal{H}$ and $\mathbb{H}_{k} p=0$ if $p \in \mathcal{L}$. Let $V$ denote the inclusion of $\mathcal{H}$ into $\mathcal{K}$. Let $X_{k}=V^{*} \mathbb{X}_{k} V$ and $H_{k}=V^{*} \mathbb{H}_{k} V$ so that $X_{k}$ and $H_{k}$ are operators on $\mathcal{H}$.

For $p, r \in \mathcal{H}$,

$$
\begin{aligned}
\left\langle X_{k} p, r\right\rangle & =\left\langle P_{x} \mathbb{X}_{k} P_{x} p, r\right\rangle \\
& =\left\langle x_{k} p, r\right\rangle \\
& =\lambda\left(r^{T} x_{k} p\right) \\
& =\lambda\left(\left(x_{k} r\right)^{T} p\right) \\
& =\left\langle p, x_{k} r\right\rangle \\
& =\left\langle p, P_{x} \mathbb{X}_{k} P_{x} r\right\rangle \\
& =\left\langle p, X_{k} r\right\rangle .
\end{aligned}
$$

Thus, each $X_{k}$ is selfadjoint and a similar argument shows that each $H_{k}$ is also selfadjoint. Further, if $r \in \mathcal{N}_{M, N}$, then $r(X, H) \emptyset=r$. Hence, if $q \in \mathcal{N}_{M, N}$ also, then

$$
<q(X, H) \emptyset, r(X, H) \emptyset>=<q, r>=\lambda\left(r^{T} q\right) .
$$


To prove $(b)$, let $p \in \mathcal{K}$ be given. There is $m \in \mathcal{H}$ and $n \in \mathcal{L}$ with $p=m+n$. Then

$$
\begin{aligned}
\left\|\mathbb{X}_{k}(p)\right\| & =\left\|\mathbb{X}_{k}(m+n)\right\| \\
& =\left\|\mathbb{X}_{k}(m)\right\| \\
& =\left\|x_{k} m\right\|
\end{aligned}
$$

and

$$
\|m\|^{2}-\left\|x_{k} m\right\|^{2}=\lambda\left(m^{T}\left(1-x_{k}^{2}\right) m\right) \geq 0
$$

as $m^{T}\left(1-x_{k}^{2}\right) m \in \mathcal{C}_{M+1, N}$ by Lemma 3.2. Hence, as $\left\|\mathbb{X}_{k}(p)\right\|=\left\|x_{k} m\right\| \leq$ $\|m\| \leq\|p\|$, each $\mathbb{X}_{k}$ is a contraction; and thus each $X_{k}$ is a contraction.

Lemma 3.5 Fix $M$ and $N$ and let d denote the dimension of $\mathcal{N}_{M, N}$.

(a) There exists a (real) Hilbert space $\mathcal{H}$ of dimension $d$, a tuple $X=$ $\left(X_{1}, \ldots, X_{g_{x}}\right)$ of selfadjoint contractions on $\mathcal{H}$, and a tuple $H=\left(H_{1}, \ldots, H_{g_{h}}\right)$ of selfadjoint contractions acting on $\mathcal{H}$ such that $\left\|X_{k}\right\|^{2} \leq \frac{1}{2}$ and $\left\|H_{k}\right\| \leq 1$ for each $1 \leq k \leq g_{x}$ and $1 \leq k \leq g_{h}$ respectively, and such that if $p \in \mathcal{N}_{M, N}$ and $p(X, H)=0$, then $p=0$.

(b) Given $M$ and $N$, there exists a linear functional $\mu: \mathcal{N}_{2 M, 2 N} \longrightarrow \mathbb{R}$ such that $\mu(p) \geq 0$ whenever $p \in \mathcal{C}_{M, N}, \mu\left(p^{T}\right)=\mu(p)$, and $\mu\left(p^{T} p\right)>0$ for all nonzero $p \in \mathcal{N}_{M, N}$. Moreover, $\mu$ is defined in terms of the trace so that $\mu(p q)=\mu(q p)$ for $p, q \in \mathcal{N}_{N, M}$.

Proof. Let $\lambda: \mathcal{N}_{2(M+1), 2(N+1)} \longrightarrow \mathbb{R}$ denote a functional from Lemma 3.3 corresponding to $(N+1, M+1)$ such that $\lambda\left(p^{T} p\right)>0$ for all nonzero $p \in$ $\mathcal{N}_{M+1, N+1}$ and let $(X, H)$ denote the tuple from Lemma 3.4 associated to $\lambda$. If $p \in \mathcal{N}_{M, N}$ and $P(X, H) \gamma=0$, then $<p(X, H) \gamma, p(X, H) \gamma>=\lambda\left(p^{T} p\right)=0$ in which case the hypothesis on $\lambda$ implies $p=0$.

Given $t>0$, let $p_{t}(x, h)=p(t x, t h)$. In particular, if $p \in \mathcal{N}_{M, N}$, then $p_{t} \in \mathcal{N}_{M, N}$ also. Further, $p=0$ if and only if $p_{t}=0$ for all $t$. Thus, by what is proved above, if $p \neq 0$, then $p_{t}(X, H) \gamma=p(t X, t H) \gamma \neq 0$. Choosing $t$ so that $\left\|t X_{k}\right\| \leq \frac{1}{2}$ and $\left\|t H_{k}\right\| \leq 1$ for all $k$ proves $(a)$.

To prove $(b)$, let $X$ denote a tuple as in part $(a)$ and define $\mu: \mathcal{N}_{2 M, 2 N} \rightarrow$ $\mathbb{R}$ by $\mu(p)=\operatorname{trace}(p(X, H))$. Then $\mu$ is linear; if $p \in \mathcal{C}_{M, N}$, then $p(X, H) \geq 0$ so that $\mu(p) \geq 0$; for $p \in \mathcal{N}_{2 M, 2 N}$, we have

$$
\mu\left(p^{T}\right)=\operatorname{trace}\left(p^{T}(X, H)\right)=\operatorname{trace}\left(p(X, H)^{T}\right)=\operatorname{trace}(p(X, H))=\mu(p) ;
$$

and if $p \in \mathcal{N}_{M, N}$ and

$$
\mu\left(p^{T} p\right)=\operatorname{trace}\left(p(X)^{T} p(X)\right)=0,
$$


then $p(X)=0$ and so, by $(a), p=0$.

Given $M$ and $N$, define norms $\|\cdot\|_{2}$ and $\|\cdot\|_{\Pi}$ on $\mathcal{N}_{M, N}$, by

$$
\left\|\sum_{w \in \mathcal{F}_{M, N}} p_{w} w\right\|_{2}^{2}=\sum\left|p_{w}\right|^{2}
$$

and

$$
\|p\|_{\Pi}=\sup \{\|p(X, H)\|:(X, H) \in \Pi\}
$$

where $\Pi$ denotes the collection of tuples $(X, H)$ where $X=\left(X_{1}, \ldots, X_{g_{x}}\right)$ and $H=\left(H_{1}, \ldots, H_{g_{h}}\right)$ are selfadjoint tuples acting on the same (real) Hilbert space and $\left\|X_{k}\right\|^{2} \leq \frac{1}{2}$ and $\left\|H_{k}\right\| \leq 1$. Observe that $\|p\|_{\Pi}$ is finite and is easily seen to be a seminorm. Item $(a)$ of Lemma 3.5 implies $\|p\|_{\Pi}=0$ if and only if $p=0$ so that $\|\cdot\|_{\Pi}$ is indeed a norm.

Lemma 3.6 Given $M$ and $N$, the norms $\|\cdot\|_{2}$ and $\|\cdot\|_{\Pi}$ are equivalent on $\mathcal{N}_{M, N}$. That is, for each $M$ and $N$ there exists positive constants $K_{M, N}^{\prime}$ and $K_{M, N}$ such that for all $p \in \mathcal{N}_{M, N}$,

$$
K_{M, N}\|p\|_{2} \leq\|p\|_{\Pi} \leq K_{M, N}^{\prime}\|p\|_{2} .
$$

Proof. Both are norms on the finite dimensional (real) vector space $\mathcal{N}_{M, N}$.

\subsection{Separating $q$ from $\mathcal{C}$}

In this section outsiders $q \notin \mathcal{C}$ are separated from $\mathcal{C}$ by a linear functional which is nonnegative on $\mathcal{C}$. The key point is that the cone $\mathcal{C}$ is closed. There is some ambiguity in representing elements in $\mathcal{C}_{M, N}$ as $V(x, h)^{T} P(x) V(x, h)$ as $P \in \mathcal{P}_{M, N}$ need not be unique. However, it is possible to bound the norm (any norm) of the entries of the $P$ which represent a given $q$.

\subsubsection{Bounded P's}

Recall the definitions of $P^{j, j}$ given before Definition 2.1 .

Lemma 3.7 Given $M$ and $N$, there exists a constant $C$ such that if $q \in$ $\mathcal{C}_{M, N}$ and if $P \in \mathcal{P}_{M, N}$ is such that $q=V_{M, N}^{T} P V_{M, N}$, then

$$
\left\|P_{\alpha, \alpha}^{j, j}\right\|_{\Pi} \leq C\left(\|q\|_{\Pi}+\left\|V_{M, N}^{T} V_{M, N}\right\|_{\Pi}\right)
$$

for each $1 \leq j \leq N$ and $\alpha \in \Gamma_{M, N}$ of degree exactly $j$ in $h$. Also, $\left\|P^{0,0}\right\|_{\Pi} \leq$ $C\left(\|q\|_{\Pi}+\left\|V_{M, N}^{T} V_{M, N}\right\|_{\Pi}\right)$. 
Proof. As for the $P^{0,0}$ term, simply observe that $P^{0,0}$ is the homogeneous of degree 0 in $h$ part of $q$. In particular, $\left\|P^{0,0}\right\|_{2} \leq\|q\|_{2}$. In view of Lemma 3.6 there is a constant $K$ such that $\left\|P^{0,0}\right\|_{\Pi} \leq K\|q\|_{\Pi}$.

Since $P(X) \geq 0$ for all selfadjoint tuples $X=\left(X_{1}, \ldots, X_{g_{x}}\right)$ of contractions, results of $[\mathrm{HM}]$ imply there exists polynomials $S_{k}$ and $R$ (matrix valued) in $x$ such that

$$
P+I=\sum_{k}\left(S_{k}\right)^{T}\left(1-x_{k}^{2}\right) S_{k}+R^{T} R,
$$

where $I$ is the identity (matrix) polynomial. Here, and in what follows $1 \leq k \leq g_{x}$. Note that the degrees of the polynomials $S$ and $R$ and the number of rows in the matrices can be quite large and it may well be that in similar representations for $P+\epsilon I, \epsilon>0$, that one or the other tends to infinity as $\epsilon$ tends to 0 .

Write

$$
S_{k}=\left(\begin{array}{llll}
S_{k}^{0} & S_{k}^{1} & \ldots & S_{k}^{N}
\end{array}\right)
$$

with respect to the same decomposition as $V=\oplus_{0}^{N} V^{j}$. Express $R$ similarly. With these notations,

$$
P^{j, j}+I=\sum_{k}\left(S_{k}^{j}\right)^{T}\left(1-x_{k}^{2}\right) S_{k}^{j}+\left(R^{j}\right)^{T} R^{j},
$$

and

$$
\left(V^{j}\right)^{T}\left(P^{j, j}+I\right) V^{j}=\left(V^{j}\right)^{T}\left(\sum_{k}\left(S_{k}^{j}\right)^{T}\left(1-x_{k}^{2}\right) S_{k}^{j}+\left(R^{j}\right)^{T} R^{j}\right) V^{j} .
$$

Evaluating (16) at tuples $X$ and $H$ of selfadjoint operators gives the inequality,

$$
\begin{aligned}
& \left(V^{j}\right)^{T}(X, H)\left(P^{j, j}(X)+I\right) V^{j}(X, H) \\
& \quad \leq\left(V^{j}\right)^{T}(X, H)\left(\sum_{k}\left(S_{k}^{j}\right)^{T}(X) S_{k}^{j}(X)+\left(R^{j}\right)^{T}(X) R^{j}(X)\right) V^{j}(X, H)
\end{aligned}
$$

Letting $V^{j}$ also denote the vector with $V^{j}$ in the $j$-th position and zero elsewhere and observing that $S_{k} V^{j}=S_{k}^{j} V^{j}$, the above inequality becomes,

$$
\begin{aligned}
& \left(V^{j}\right)^{T}(X, H)(P(X)+I) V^{j}(X, H) \\
& \quad \leq \sum_{k}\left(S_{k} V^{j}\right)^{T}(X, H)\left(S_{k} V^{j}\right)(X, H)+\left(R^{j} V^{j}\right)^{T}(X)\left(R^{j} V^{j}\right)(X) .
\end{aligned}
$$


Similarly, evaluating (13) at $X$ and $H$, tuples of symmetric operators, where $\left\|X_{k}\right\|^{2} \leq \frac{1}{2}$ produces the inequality,

$$
\begin{aligned}
& V(X, H)^{T}(P(X)+I) V(X, H) \\
& \quad \geq V(X, H)^{T}\left(\frac{1}{2}\left(\sum_{k}\left(S_{k}\right)^{T}(X) S_{k}(X)\right)+R^{T}(X) R(X)\right) V(X, H) .
\end{aligned}
$$

Define

$$
Q=\left(\begin{array}{c}
\left(\frac{1}{2}\right)^{\frac{1}{2}} S_{1} V \\
\left(\frac{1}{2}\right)^{\frac{1}{2}} S_{2} V \\
\vdots \\
\left(\frac{1}{2}\right)^{\frac{1}{2}} S_{g_{x}} V \\
R V
\end{array}\right)
$$

To save space, we will abbreviate columns using the $\bigoplus$ notation; for example, $Q=\bigoplus_{k}\left(\frac{1}{2}\right)^{\frac{1}{2}}\left(S_{k} V\right) \bigoplus R V$. Thus

$$
\begin{gathered}
Q^{T}(X, H) Q(X, H)= \\
V(X, H)^{T}\left(\frac{1}{2}\left(\sum_{k}\left(S_{k}\right)^{T}(X) S_{k}(X)\right)+R^{T}(X) R(X)\right) V(X, H)
\end{gathered}
$$

which is the right hand side of (19). In particular, if $\left\|X_{k}\right\|^{2} \leq \frac{1}{2}$, then, for real $t$,

$$
\begin{aligned}
Q(X, t H)^{T} Q(X, t H) & \leq V(X, t H)^{T}(P(X)+I) V(X, t H) \\
& =q(X, t H)+V(X, t H)^{T} V(X, t H) .
\end{aligned}
$$

Thus,

$$
\begin{aligned}
\|Q(X, t H)\|^{2} & =\left\|Q(X, t H)^{T} Q(X, t H)\right\| \\
& \leq\|q(X, t H)\|+\left\|V^{T}(X, t H) V(X, t H)\right\| .
\end{aligned}
$$

Hence, for $\left\|X_{k}\right\|^{2} \leq \frac{1}{2},\left\|H_{k}\right\| \leq 1$ and $|t|<1$,

$$
\|Q(X, t H)\| \leq\left(\|q\|_{\Pi}+\left\|V^{T} V\right\|_{\Pi}\right)^{\frac{1}{2}}
$$

Let $Q_{j}=\left(\bigoplus_{k}\left(\frac{1}{2}\right)^{\frac{1}{2}} S_{k}^{j} V^{j}\right) \bigoplus R^{j} V^{j}$. Then,

$$
\begin{gathered}
2 Q_{j}(X, H)^{T} Q_{j}(X, H) \geq \\
\sum_{k}\left(S_{k}^{j} V^{j}\right)^{T}(X, H) S_{k}^{j} V^{j}(X, H)+\left(R^{j} V^{j}\right)^{T}(X, H) R^{j} V^{j}(X, H) .
\end{gathered}
$$


Since the right hand side above is the same as the right hand side of (18),

$$
2 Q_{j}(X, H)^{T} Q_{j}(X, H) \geq V^{j}(X, H)^{T} P^{j, j}(X) V^{j}(X, H)
$$

so that

$$
\left\|Q_{j}(X, H)\right\|^{2} \geq \frac{1}{2}\left\|V^{j}(X, H)^{T} P^{j, j}(X) V^{j}(X, H)\right\| .
$$

For each $0 \leq j \leq N$ there exists a polynomial $\gamma_{j}(t)$ (an old fashion polynomial in the real variable $t$ ) of degree at most $N$ such that $\int_{0}^{1} t^{j} \gamma_{j}(t) d t=1$ and for each $0 \leq k \neq j \leq N, \int_{0}^{1} t^{k} \gamma_{j}(t) d t=0$. Consequently,

$$
\begin{aligned}
\int_{0}^{1} Q(X, t H) \gamma_{j}(t) d t & =\bigoplus_{k} \int_{0}^{1}\left(\frac{1}{2}\right)^{\frac{1}{2}} S_{k} V(X, t H) \bigoplus R V(X, t H) \gamma_{j}(t) d t \\
& =\left(\bigoplus_{k}\left(\frac{1}{2}\right)^{\frac{1}{2}} S_{k}^{j}(X) V^{j}(X, H)\right) \bigoplus R^{j}(X) V^{j}(X, H) \\
& =Q_{j}(X, H) .
\end{aligned}
$$

Thus, for $(X, H) \in \Pi$ (where $\Pi$ is defined near (12))

$$
\left\|Q_{j}(X, H)\right\| \leq \int_{0}^{1}\|Q(X, t H)\|\left|\gamma_{j}(t)\right| d t \leq\left\|\gamma_{j}\right\|_{\infty}\left(\|q\|_{\Pi}+\left\|V^{T} V\right\|_{\Pi}\right)^{\frac{1}{2}}
$$

where $\left\|\gamma_{j}\right\|_{\infty}$ is the supremum norm of $\gamma_{j}$ in the interval $0 \leq t \leq 1$ and (23) was used in the second inequality. Combining (27) and (26) gives,

$$
\left\|V^{j}(X, H) P^{j, j}(X) V^{j}(X, H)\right\| \leq 2\left\|\gamma_{j}\right\|_{\infty}^{2}\left(\|q\|_{\Pi}+\left\|V^{T} V\right\|_{\Pi}\right) .
$$

Thus, if we let $C=2 \max \left\{\left\|\gamma_{j}\right\|_{\infty}^{2}: 1 \leq j \leq N\right\}$, then

$$
\left\|V^{j}(X, H) P^{j, j}(X) V^{j}(X, H)\right\| \leq C\left(\|q\|_{\Pi}+\left\|V^{T} V\right\|_{\Pi}\right)
$$

for all $(X, H) \in \Pi$ and all $1 \leq j \leq N$.

To complete the proof, notice that

$$
\left(V_{M, N}^{j}\right)^{T} P^{j, j} V_{M, N}^{j}=\sum \alpha^{T} h_{k} P_{h_{k} \alpha, h_{\ell} \beta}^{j, j} h_{\ell} \beta,
$$

where the sum is over all $\alpha, \beta$ with degree exactly $j-1$ in $h$ (and at most $M$ in $x)$ and $1 \leq k, \ell \leq g_{h}$. By Lemma 3.1, for distinct $\left(h_{k} \alpha, h_{m} \beta\right)$ the terms $\beta^{T} h_{\ell} P_{h_{k} \alpha, h_{\ell} \beta}^{j, j} h_{k} \alpha$ are $\|\cdot\|_{2}$ orthogonal. Since also, for each relevant $k, \ell, \alpha, \beta$, $\left\|\alpha^{T} h_{k} P_{h_{k} \alpha, h_{\ell} \beta}^{j, j} h_{\ell} \beta\right\|_{2}=\left\|P_{h_{k} \alpha, h_{\ell} \beta}^{j, j}\right\|_{2}$, it follows that

$$
\left\|V^{j}(M, N)^{T} P^{j, j} V^{j}(M, N)\right\|_{2}^{2}=\sum\left\|P_{h_{k} \alpha, h_{\ell} \beta}^{j, j}\right\|_{2}^{2} .
$$

Since the norms $\|\cdot\|_{2}$ and $\|\cdot\|_{\Pi}$ are equivalent on $\mathcal{N}_{2 M, 2 N}$, the lemma follows by combining (31) and (29). 


\subsubsection{The cone $\mathcal{C}_{M, N}$ is closed}

Lemma 3.8 Given $M$ and $N$ and a bounded set $S \subset \mathcal{C}_{M, N}$, there exists a $C_{S}$ such that if $q \in S$ and if $P \in \mathcal{P}_{M, N}$ is such that $q=V_{M, N}^{T} P V_{M, N}$, then $\left\|P_{\alpha, \beta}^{j, k}\right\|_{\Pi} \leq C_{S}$ for each $0 \leq j, k \leq N$ and $\alpha, \beta \in \Gamma_{M, N}$ of degree exactly $j$ in $h$.

Proof. Let $C$ be as in Lemma 3.7 and let $K$ denote a bound for $S$ so that if $q \in S$, then $\|q\|_{\Pi} \leq K$. Then, $\left\|P_{\alpha, \alpha}^{j, j}\right\| \leq C\left(K+\left\|V^{T} V\right\|_{\Pi}\right)$ for all relevant $P$ and admissible choices of $j$ and $\alpha$. Since $P(X) \geq 0$ for any tuple $X$ of selfadjoint contractions, it follows that the off diagonal entries of $P$ also satisfy the inequality $\left\|P_{\alpha, \beta}^{j, k}(X)\right\| \leq C\left(K+\left\|V^{T} V\right\|_{\Pi}\right)$ for any tuple $X$ of selfadjoint contractions. The lemma now follows.

Proposition 3.9 For each $M$ and $N$, the cone $\mathcal{C}_{M, N}$ is a closed subset of $\mathcal{N}_{2 M, 2 N}$.

Proof. Suppose $q_{n} \in \mathcal{C}_{M, N}$ converges to $q$. Then $\left\|q_{n}\right\|_{\Pi}$ is a bounded sequence and there is a $K$ such that $\left\|q_{n}\right\|_{\Pi}+\left\|V^{T} V\right\|_{\Pi} \leq K$.

For each $n$ there exists $P(x ; n) \in \mathcal{P}_{M, N}$ such that $q_{n}=V^{T} P(\cdot ; n) V$. Thus, by Lemma 3.8 , there is a constant $C$ such that which bounds the $\|\cdot\|_{\Pi}$ norm of all the entries of all the $P(x ; n)$. Since the entries of each $P(x ; n)$ have degree at most $2 M$, it follows that some subsequence of $P(x ; n)$, still denoted $P(x ; n)$, converges to some $P$ and therefore, for any tuple $X$, $P(X ; n)$ converges to $P(X)$. In particular, $P$ satisfies item (1) of Definition 2.1. Similarly, as each $P(x ; n)$ satisfies items $(2)$ and $(3)$ of Definition 2.1, so does $P$. Thus $P \in \mathcal{P}_{M, N}$. Finally, as $P(x ; n)$ converges entry-wise, $V^{T} P(\cdot ; n) V$ converges to $V^{T} P V$.

\subsubsection{The separation argument}

Proposition 3.10 Fix $M$ and $N$. If $q \in \mathcal{N}_{2 M, 2 N}$, but $q \notin \mathcal{C}_{M, N}$, then there exists a linear functional $\lambda: \mathcal{N}_{2 M, 2 N} \longrightarrow \mathbb{R}$ such that $\lambda\left(p^{T}\right)=\lambda(p)$, $\lambda\left(C_{M, N}\right) \geq 0$, and $\lambda\left(p^{T} p\right)>0$ for all nonzero $p \in \mathcal{N}_{M, N}$, but $\lambda(q)<0$. In particular, $\lambda(\emptyset)>0$.

Proof. By Proposition 3.9, $\mathcal{C}_{M, N} \subset \mathcal{N}_{2 M, 2 N}$ is a closed set. Since $\mathcal{C}_{M, N}$ is a closed convex set and $q \notin \mathcal{C}_{M, N}$, there exists a (real) linear functional $\Lambda: \mathcal{N}_{2 M, 2 N} \longrightarrow \mathbb{R}$ and a real number $c$ so that $\Lambda(q)<c<\Lambda(p)$ for all $p \in \mathcal{C}_{M, N}$. Since $\mathcal{C}_{M, N}$ is a cone containing 0 , it follows that $c \leq 0$. Define 
$\Lambda^{s}(p)=\frac{1}{2}\left(\Lambda\left(p+p^{T}\right)\right)$. Then $\Lambda^{s}$ is linear; $\Lambda^{s}\left(p^{T}\right)=\Lambda^{s}(p)$; and $\Lambda^{s}(p)=\Lambda(p)$ if $p$ is symmetric and thus $\Lambda^{s}(p) \geq 0$ for all $p \in \mathcal{C}_{M, N}$ and $\Lambda(q)<0$.

Let $\mu$ denote the linear functional of Lemma $3.5(b)$. There is a $\kappa>0$ such that $\Lambda^{s}(q)+\kappa \mu(q)<0$. Let $\lambda=\Lambda^{s}+\kappa \mu$. Then $\lambda(q)<0, \lambda\left(\mathcal{C}_{M, N}\right) \geq 0$, and $\lambda\left(p^{T} p\right)>0$ for all nonzero $p \in \mathcal{N}_{M, N}$.

\section{Proof of the Theorem 2.2}

Let $q \in \mathcal{N}_{M, N} \subset \mathcal{N}_{2(M+1), 2(N+1)}$ be given. If $q \notin \mathcal{C}_{M+1, N+1}$, then there is a linear functional $\lambda$ as in Proposition 3.10 with $(M+1, N+1)$ in place of $(M, N)$. Let $\mathcal{H}, \gamma$, and $(X, H)$ be as in Lemma 3.4 corresponding to $\lambda$. In particular, $X$ and $H$ are selfadjoint tuples, each $X_{k}$ is a contraction, and $<q(X, H) \gamma, \gamma>=\lambda(q)<0$. This proves the contrapositive; i.e., if $q \notin \mathcal{C}$, then there are tuples $X$ and $H$ with the right properties such that $q(X, H)$ is not positive semidefinite. Indeed, it shows more. If $q \in \mathcal{N}_{M, N}$ and $q(X, H) \geq 0$ for all relevant $(X, H)$, then $q \in \mathcal{C}_{M+1, N+1}$.

To prove the stronger conclusion of the theorem, suppose now that $q \in$ $\mathcal{N}_{M, 2 N}$. Then from what is already proved, $q \in \mathcal{C}_{M+1,2 N+1}$. Thus, there exists a $P \in \mathcal{P}_{M+1,2 N+1}$ so that $q=V^{T} P V$. For a given $(X, H)$ consider

$$
\begin{aligned}
0=\lim _{t \rightarrow \infty} \frac{1}{t^{2(2 N+1)}} q(X, t H) & =\lim _{t \rightarrow \infty} V(X, t H)^{T} P(X) V(X, t H) \\
& =V^{2 N+1}(X, H)^{T} P^{2 N+1,2 N+1}(X) V^{2 N+1}(X, H)
\end{aligned}
$$

from which it follows that $V^{2 N+1}(X, H)^{T} P^{2 N+1,2 N+1}(X) V^{2 N+1}(X, H)=0$ for all $(X, H)$. From a version of Lemma 3.5(a), it follows that

$$
V^{2 N+1}(x, h)^{T} P^{2 N+1,2 N+1}(x) V^{2 N+1}(x, h)=0 .
$$

By Lemma $3.1, P^{2 N+1,2 N+1}=0$. Since $P(X) \geq 0$ for all tuples of contractions and $P^{2 N+1,2 N+1}(X)=0$, it must be the case that $P^{2 N+1, j}(X)=$ $P^{j, 2 N+1}(X)=0$ for each $0 \leq j \leq 2 N$. Thus $P^{2 N+1, j}=P^{j, 2 N+1}=0$ for each $j$ and hence $q \in \mathcal{C}_{M, 2 N+1-1}$. Continuing by induction completes the proof.

\section{References}

[A1] J. Agler, An abstract approach to model theory, Surveys of some recent results in operator theory, Vol. II, 1-23, Pitman Res. Notes Math. Ser., 192, Longman Sci. Tech., Harlow, 1988. 
[A2] J. Agler, On the representation of certain holomorphic functions defined on a polydisk, Topics in operator theory: Ernst D. Hellinger memorial volume, 47-66, Oper. Theory Adv. Appl., 48, Birkhuser, Basel, 1990

[AP] A. Arias and G. Popescu, Factorization and reflexivity on Fock spaces Integral Equations Operator Theory 23 (1995), no. 3, 268-286.

[BCR] J. Bochnack, M. Coste and J.-F. Roy, Géométrie Algébrique Réele, Springer, New York, 1987.

[CHSY] J.F. Camino, J.W. Helton, R.E. Skelton, J. Ye, Matrix Inequalities: A Symbolic Procedure to

Determine Convexity Automatically To appear IEOT July 2003

[DP] K.R. Davidson and D.R. Pitts, The algebraic structure of noncommutative analytic Toeplitz algebras, Math. Ann. 311 (1998), no. 2, 275-303.

[H] J.W. Helton, Positive non commutative polynomials are sums of squares Ann. Math (to appear).

[HM] J.W. Helton and S. McCullough, A Positivstellensatz for Noncommutative Polynomials TAMS (to appear).

[HM2] J.W. Helton and S. McCullough, Convex noncommutative polynomials have degree two or less, preprint.

[HMP] J.W. Helton, M. Putinar, S. McCullough, A noncommutative Positivstellensatz on isometries, preprint.

[M] S. McCullough, Factorization of operator valued polynomials in several noncommuting variables, Linear Algebra Appl. 326 (2001) no. 1-3, 193203.

[MP] S. McCullough and M. Putinar, Noncommutative Sums of Squares, preprint.

[PD] A.Prestel and C.N.Delzell, Positive Polynomials, Springer, Berlin, 2001.

[PS] C. Procesi and M. Schacher, A noncommutative real Nullstellensatz and Hilbert's 17th problem, Ann. of Math. (2) 104 (1976), no. 3, 395-406.

[PV] M. Putinar and F.-H. Vasilescu, Solving moment problems by dimensional extension, Ann. Math. 149 (1999), 1087-1107. 
[RS] M. Reed and B. Simon, Methods of Modern Mathematical Physics Vol. 1: Functional Analysis, Academic Press, San Diego, 1980.

[R] B. Reznick, Sums of even powers of real linear forms, Mem. Amer. Math. Soc. 96 (1992) No. 463, mer. Math. Soc., Providence, R.I.

[St] G. Stengle, A Nullstellensatz and a Positivstellensatz in semialgebraic geometry, Math. Ann. 289(1991), 203-206.

[Sc] K. Schmüdgen, On the moment problem of closed semialgebraic sets, J. reine angew. Math., to appear. 


\section{NOT FOR PUBLICATION}

\section{Contents}

1 Introduction 2

2 Notation and Main Result 5

2.1 Notation . . . . . . . . . . . . . . . 5

2.2 Main result . . . . . . . . . . . . . . . . 7

3 Components of the proof $\quad 8$

3.1 Some first words about words . . . . . . . . . . . 8

3.2 Positive functionals, tuples, and the openness condition . . . 9

3.3 Separating $q$ from $\mathcal{C} \ldots \ldots \ldots \ldots 12$

3.3 .1 Bounded $P^{\prime}$ s . . . . . . . . . . . . . 12

3.3.2 The cone $\mathcal{C}_{M, N}$ is closed . . . . . . . . . . 16

3.3.3 The separation argument . . . . . . . . . 16

4 Proof of the Theorem 2.2 17 